\title{
Autowaves of component concentration under directed crystallization
}

\author{
A. P. Gus'kov \\ Institute of Solid State Physics Academy of Sciences of Russia, \\ Moscow District, Chernogolovka, 142432, Russia \\ E-mail: gusskov@issp.ac.ru
}

\begin{abstract}
The occurrence of autowaves at the interface as a result of a first-order phase transition had been examined. The linear analysis of a system of the equations for a liquid which contains two components shows that a phase transition at the interface can give rise to autowaves. A spectrum of these autowaves depends on parameters of the system. For a special case of the system parameters an analysis is made of the dependence of the spectrum of autowaves from a temperature gradient the interface. We show that as a result of a interface instability in the bulk solid phase there are various periodic structures of distribution of the component concentration. Surfaces of equal concentration for the spectra of the autowaves are built. A qualitative and quantitative comparison of the numerical calculations with experimental data is made.
\end{abstract}

PACS: 61.72. Ss, $66.30 .-$ h

\section{Introduction}

We have shown [1-3], that a first-order phase transition can give rise to various types of autowaves of a component concentration at the interface. We obtained a dependence of the change in the spectrum of the autowaves on the temperature gradient at the interface.

The autowaves of the component concentration form a nonuniform distribution of the component in the solid phase as a result of the crystallization. Modes of the autowaves define a structure of the component distribution. Spatial period of the autowaves defines characteristic size of this structure. In this study we analyze of the distribution of the component concentration in the bulk solid phase and consider the types of autowaves, which were obtained in Ref. 3.

\section{Formulation of the problem}

Let $T(y, z, \tau)$ be the temperature normalized to the phase transition temperature $T_{e 0}$ and to the initial component concentration $C_{0} ; C(y, z, \tau)$ is the component concentration normalized to the initial concentration; $y, z, \tau$ are the dimensionless coordinates and the time: $y=\alpha y_{r}, z=\alpha z_{r}$, $\tau=\alpha^{2} \chi_{0} \tau_{r} ; D$ is the dimensionless factor of diffusion in a melt, $D=D_{r} / \chi_{0} ; \chi=\chi_{r} / \chi_{0}$ is the factor of thermal diffusivity; $\varepsilon$ is the heat of phase transition normalized to the specific heat capacity and the temperature of phase transition; $y_{r}, z_{r}, \tau_{r}, D_{r}, \chi_{r}$ and $\varepsilon_{r}$ are dimensional quantities, $\chi_{0}=10^{-5} \mathrm{~m}^{2} / \mathrm{s}$, and $\alpha=10^{2} \mathrm{~m}^{-1}$. Let the conditions of melt cooling be such that the flat front of crystallization in a stationary regime is moving at a constant velocity $V_{s}$.

We take into account the heat conduction in the solid and liquid phases and diffusion of the component in the liquid phase. To reduce the calculations in the equations, we do not write the coordinate $x$. The values relating to the solid phase are designated by a prime. In the coordinates $y, z$, $\tau$, which are rigidly connected with the moving front, the original system of equations has the form

$$
\begin{gathered}
\frac{\partial T^{\prime}}{\partial \tau}=\chi^{\prime} \Delta T^{\prime}+V \frac{\partial T^{\prime}}{\partial z} ; \quad z_{0} \leq z \leq 0 ; \\
\frac{\partial T}{\partial \tau}=\chi \Delta T+V \frac{\partial T}{\partial z} ; \quad 0 \leq z<\infty ; \\
\frac{\partial C}{\partial \tau}=D \Delta C+V \frac{\partial C}{\partial z} ; \quad 0 \leq z<\infty ; \\
\left.\chi^{\prime} \frac{\partial T^{\prime}}{\partial z}\right|_{z=-0}-\left.\chi \frac{\partial T}{\partial z}\right|_{z=+0}=\varepsilon V ;
\end{gathered}
$$




$$
\begin{gathered}
\left.\chi \frac{\partial T}{\partial z}\right|_{z=-0}=I ; T(0)=T^{\prime}(0) ; T(\infty)=T_{00} ; \\
\left.D \frac{\partial C}{\partial z}\right|_{z=0}=V(1-k) C(0) ; C(\infty)=1 ; \\
V=V(T(y, 0, \tau), C(y, 0, \tau)) .
\end{gathered}
$$

Here

$$
k=\frac{C_{\infty}}{C_{s}(0)},
$$

where $C_{s}(0)$ is the component concentration at the interface in the stationary regime.

To study the stability of the stationary solutions against small perturbations of the temperature and concentration, we seek the solutions as

$$
\begin{gathered}
T^{\prime}=T_{s}^{\prime}(z)+T_{m}^{\prime}(z) \exp (\omega \tau+K y) ; \\
T=T_{s}(z)+T_{m}(z) \exp (\omega \tau+K y) ; \\
C=C_{s}(z)+C_{m}(z) \exp (\omega \tau+K y) ; \omega=\omega_{1}+i \omega_{2} ; \\
K=K_{1}+i K_{2}, T_{m}^{\prime}(z)<<T_{s}^{\prime}(z) ; T_{m}(z)<<T_{s}(z) ; \\
C_{m}(z)<<C_{s}(z),
\end{gathered}
$$

where $T_{s}^{\prime}(z), T_{s}(z)$, and $C_{s}(z)$ are the solutions of the stationary problem. We linearize the boundary value problem to small $T_{m}^{\prime}(z), T_{m}(z), C_{m}(z)$ quantities and find the solutions of the stationary problem and for small perturbations of the problem. The homogeneous boundary value problem for small perturbations gives the dispersion relation.

The dispersion relation contains all the parameters that are contained in the boundary value problem. These parameters depend on the composition of the material and on the external conditions of crystallization. They are reduced to six dimensionless parameters:

$$
\begin{gathered}
\mu_{1}=\frac{\chi}{D} ; \quad \mu_{2}=\frac{\chi}{\chi^{\prime}} ; \quad P_{1}=\frac{\gamma}{V_{s}} ; \quad P_{2}=\frac{2 \varepsilon \theta}{V_{s}} ; \\
\Phi_{0}=\frac{I_{0}}{\varepsilon V_{s}}=\frac{\chi}{\varepsilon V_{s}} \operatorname{grad} T_{s}(0) .
\end{gathered}
$$

The solution of this task resulted in work in Ref. 3. To find the spectrum of the autowaves we used the maximum-growth-rate hypothesis.

\section{Distribution of the component concentration in the solid phase}

Let us consider the results form the physical point of view. According to the examined theory, the formation of the structure of the component distribution begins in the liquid in front of the interface. The liquid phase has a homogeneous distribution of the component at a sufficiently large distance from the interface. The component concentration becomes inhomogeneous near the interface as a result of nonlinear processes at the interface. It depends on both time and spatial coordinates. The inhomogeneity of the component concentration increases with decreasing distanse to the interface. As a result, the component concentration is a flat periodic structure at the interface. Upon moving the interface, this structure is fixed into the solid phase. Thus, the volumetric periodic structure of the distribution components are formed in the solid phase.

Let $k=2, \mu_{1}=500, \mu_{2}=1.5, P_{1}=-206$, and $P_{2}=-4600$. In this case the dispersion relation can be presented as the dependence in Fig. 1. Here $\operatorname{grad} T_{S}(0)$ is the temperature gradient at the interface; $L$ is a spatial period of the temporary oscillations and of the spatial distortions of the distribution of the component concentration in the solid phase, which are obtained from the expressions

$$
\begin{gathered}
L_{p}=\frac{8 \pi D}{\alpha V_{s} \Omega} ; \quad L_{d}=\frac{4 \pi D}{\alpha V_{s} \sqrt{Y}} ; \quad \Omega=\frac{4 D \omega_{1}}{V_{s}^{2}} ; \\
Y=\frac{4 D^{2} K_{2}^{2}}{V_{s}^{2}} .
\end{gathered}
$$

Here $L_{p}$ is the spatial period of the temporary oscillations, and $L_{d}$ is the period of the spatial

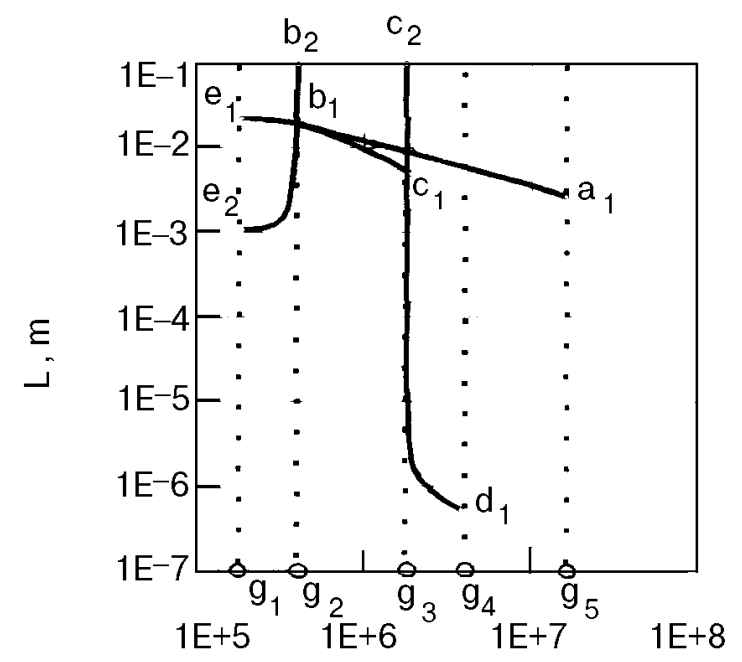

Fig. 1. Dependence of the spatial distortions $L_{d}$ and spatial period of temporary pulsations $L_{p}$ on the temperature gradient $\operatorname{grad} T_{s}(0)$ 
Fig. 2. Structure of distribution of the component concentration as grainy.

distortions. In the range $0<\operatorname{grad} T_{s}(0)<g_{1}$ and $g_{5}<\operatorname{grad} T_{s}(0)<\infty$ the system is steady. In the ranges $g_{1}<\operatorname{grad} T_{s}(0)<g_{2}$ and $g_{3}<\operatorname{grad} T_{s}(0)<g_{4}$ the system is unstable and the autowaves at the interface have one mode of the temporary oscillations and one mode of the spatial distortions. At $g_{2}<\operatorname{grad} T_{s}(0)<g_{3}$ the autowaves have two spatial modes. At $g_{4}<\operatorname{grad} T_{s}(0)<g_{5}$ the autowaves have one spatial mode.

Let us consider the distribution of the component concentration in the solid phase for each of these modes of the autowaves. In a common case for a regime with one mode of the temporary oscillations and two modes of the spatial distortions, it is possible to write the dependence of the component concentration on $x, y$, and $\tau$ at the interface as

$$
\begin{aligned}
& c(x, y, \tau)=c_{s}+\left[A_{1} \cos \left(K_{21} x\right) \cos \left(K_{21} y\right)+\right. \\
& \left.+A_{2} \cos \left(K_{22} x\right) \cos \left(K_{22} y\right)\right] \sin \left(\omega_{2} \tau\right) .
\end{aligned}
$$

Let us define the coordinate $z$ in the laboratory system of coordinates as

$$
\begin{gathered}
z=V_{s} \tau-\frac{1}{\omega_{2}}\left[A_{1} \cos \left(K_{21} x\right) \cos \left(K_{21} y\right)+\right. \\
\left.+A_{2} \cos \left(K_{22} x\right) \cos \left(K_{22} y\right)\right] \cos \left(\omega_{2} \tau\right),
\end{gathered}
$$

where $A_{1}$ and $A_{2}$ are the constant factors, and $K_{21}$, and $K_{22}$ are the wave numbers of the first and second modes.

Let us consider a surface for a constant value of the component concentration in the solid phase at the ranges $g_{1}<\operatorname{grad} T_{s}(0)<g_{2}$ and $g_{3}<\operatorname{grad} T_{s}(0)<g_{4}$. To find the surface $c(x, y, \tau)=$ const $=Q$ from Eqs. (1) and (2), we find $\tau$ in Eq. (1) and substitute it in Eq. (2). As a result, we obtain the dependence $z(x, y, \tau)$, which is at the required surface $c(x, y, \tau)=$ const $=Q$ in coordinates $(x, y, z)$. To obtain the surface $c=$ const for autowaves with one temporary mode and one spatial mode, let us assume $A_{2}=0$. For example at $Q=0.9, c_{s}=1, V_{s}=1$, $A_{1}=0.2, A_{2}=0, \omega_{2}=1, K_{21}=1$. The surfaces of the component concentration for these values of the parameters are shown in Fig. 2. They correspond to a grainy component distribution. The grains can be elongate or flattened, depending on the ratio between the initial parameters. For example, if $K_{2}<<\omega_{2}$, in the experiment, there will be a striated component distribution in the solid phase. This case corresponds to the range $g_{3}<\operatorname{grad} T_{s}(0)<g_{4}$.

In the ranges $g_{4}<\operatorname{grad} T_{s}(0)<g_{5}$ and $g_{2}<\operatorname{grad} T_{s}(0)<g_{3}$ Eq. (1) has the form

$$
\begin{gathered}
c(x, y)=c_{s}+A_{1} \cos \left(K_{21} x\right) \cos \left(K_{21} y\right)+ \\
+A_{2} \cos \left(K_{22} x\right) \cos \left(K_{22} y\right)
\end{gathered}
$$

i.e., in this case for any value of $z$ the distribution of the component concentration is the solution of Eq. (3).

Let us assume that in the range $g_{4}<\operatorname{grad} T_{s}(0)<g_{5}$ the parameters have the values $Q=0.8, \quad c_{s}=1, \quad V_{s}=1, \quad A_{1}=0.3, \quad A_{2}=0, \quad$ and $K_{21}=1$. The surfaces $c=$ const for this case are shown in Fig. 3. They represent columns which are parallel to the $z$ axis.

In the range $g_{2}<\operatorname{grad} T_{s}(0)<g_{3}$ we use the values $Q=0.7, c_{S}=1, V_{s}=1, A_{1}=0.1, A_{2}=0.3$, $K_{21}=1$, and $K_{22}=6$. The surfaces $c=$ const for

Fig. 3. Column structure distribution of the component concentration. 
Fig. 4. Structure distribution of the component concentration as columns settle down by the groups.

this case are shown in Fig. 4. They also represent the columns parallel to the $z$ axis. In contrast to the previous case, however, these columns are arranged by the groups located periodically at a certain distance between these groups.

\section{Comparison with experiment}

We did not apply this model to calculations of the phase transitions of systems with a low temperature. We want to demonstrate that in the problems where the phase transitions together with the diffusion are examined, there can be complex periodic structures. We used the maximum-growth-rate hypothesis to investigate this problem. It is not a strict mathematical method of investigating the stability problems. However, in this case it gives not only qualitatively correct results, but also quantitative agreement of the numerical calculations with experiments. We used this theory to investigate crystallization of materials at usual temperatures and obtained complete qualitative agreement with the experimental data. All the structures found above coincide with the structures of the impurity distribution of real crystal materials. The grains similar to a sphere are a grainy structure, which is formed under the crystallization of large ingots and microcrystal materials. In the same materials the elongated grains may be formed. Such a

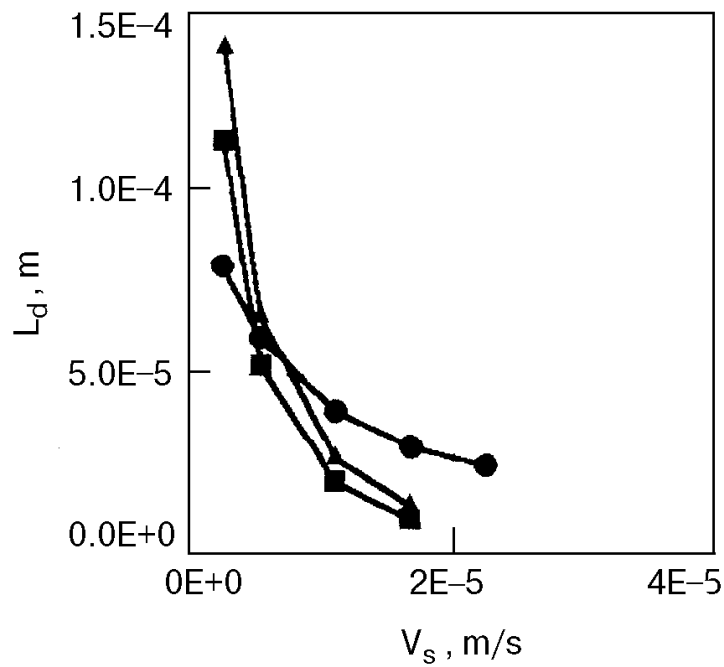

Fig. 5. Dependence of the distance between the columns of eutectic composites on the velocity of a moving interface. (0) experimental data; $(\boldsymbol{\Lambda})-$ numerical calculations at $c_{\infty}<c_{\text {eut }}$; (ם) - numerical calculations at $c_{\infty}>c_{\text {eut }}$.

structure is called as columnar crystals. The formation of the striated impurity distribution is known as crystal growth. The impurity distribution as columns (Figs. 3 and 4) is formed as a result of crystallization of eutectic composites.

Recently we have applied this theory to a real experiment. The experiments performed in our laboratory of M. Starostin gave the dependence of distance between columns of eutectic composites on the velocity of a moving interface [4]. We have calculated this dependence using the model given here. The results of calculations are in good agreement with the experimental dependences. Figure 5 shows experimental and theoretical dependences [5].

This work was supported by the Russian Foundation for Basic Research, Grant N 97-02-16845.

1. A. P. Gus'kov, in: Instabilities in Multiphase Flows, G. Gouesbet and Berlemont (eds.), Pergamon, New York (1993), p. 25.

2. A. P. Gus'kov, in: Hydromechanics and Heat and Mass Transfer in microgravity, Gordon and Breach Science Publishers, Amsterdam (1992).

3. A. P. Gus'kov, Doklady of the Russian Academy of Sciences (in Russia) 349, 561 (1996).

4. V. A. Borodin, M. Yu. Starostin, and T. N. Yalovets, J. Crystal Growth 104, 1, 143 (1990).

5. A. Gus'kov and M. Starostin, J. Mater. Science (to be published). 